\title{
Effective Resistance of a Two Layer Mesh
}

\author{
Selçuk Köse, Student Member, IEEE, and Eby G. Friedman, Fellow, IEEE
}

\begin{abstract}
The effective resistance of a resistive mesh is a commonly used analogy for various scientific and engineering problems such as $I R$ voltage drop estimation, distributed control including time synchronization and sensor localization, determining the chemical distance among multiple bonds, and finding the distance between two vertices in a graph. Resistive networks are a commonly used structure in electronics to model different elements of an integrated circuit, such as a physical substrate, an integrated circuit layout, and a power distribution network. On-chip power and ground networks are composed of orthogonal metal lines from different metal layers, and a resistive mesh is typically used to model these networks. A two layer mesh is therefore commonly used to analyze $I R$ voltage drops and decoupling capacitor placement. A closed-form expression is described here for the effective resistance between the intersections of a two layer resistive mesh where the horizontal and vertical unit resistances are different. The physical distance between the nodes of interest and the ratio between the horizontal and vertical resistances $k$ are included in the expression. The maximum error of the closed-form expression, as compared with the exact solution, is less than $\mathbf{5 \%}$ for a wide range of $k$. The error further decreases with greater separation between the nodes of interest.
\end{abstract}

Index Terms-Closed-form solution, effective resistance, noise analysis, power grid, power/ground network.

\section{INTRODUCTION}

A RESISTIVE mesh structure can be considered as an undirected graph where the weights associated with the edges are determined by the resistance between adjacent nodes within the mesh. An on-chip power and ground distribution network is commonly modeled as a resistive mesh structure with different vertical and horizontal unit resistances, as shown in Fig. 1(a) [1]-[4], where the thickness and width of the metal lines are typically different in orthogonal metal layers. Power and ground networks are illustrated in Fig. 1(a) with, respectively, dark and gray lines. A mesh structured power network and the corresponding resistive circuit model are illustrated, respectively, in Fig. 1(b) and (c). Since the power and ground distribution networks exhibit similar characteristics, only the power network is considered in this brief. This approach can also be used to determine the effective resistance in any two layer mesh structure with different horizontal and vertical unit resistances.

Manuscript received December 21, 2010; revised May 11, 2011; accepted August 26, 2011. Date of current version November 23, 2011. This work was supported in part by the National Science Foundation under Grant CCF0811317 and Grant CCF-0829915, by grants from the New York State Office of Science, Technology and Academic Research to the Center for Advanced Technology in Electronic Imaging Systems; and by grants from Intel Corporation and Qualcomm Corporation. This paper was recommended by Associate Editor K. Chakrabarty.

The authors are with the Department of Electrical and Computer Engineering, University of Rochester, Rochester, NY 14627 USA (e-mail: kose@ece. rochester.edu; friedman@ece.rochester.edu).

Digital Object Identifier 10.1109/TCSII.2011.2168016
The effective resistance of a mesh is used in power grid analysis [5], [6], substrate analysis [7], decoupling capacitance allocation [8]-[11], power dissipation [12] and electrostatic discharge (ESD) analysis [12], and measuring resistance variations in power distribution networks [13]. The effective resistance is used to determine the effective region of a decoupling capacitor [8], [14]. For instance, the effective resistance between hot spots and available white spaces in a circuit floorplan provides a means to evaluate the effectiveness of a decoupling capacitor placed at different locations. A lower effective resistance between a hot spot and a decoupling capacitor leads to a faster response time for the decoupling capacitor. Additionally, the effective resistance between a decoupling capacitor and a power supply connection provides an estimate of the recharge time of the capacitor. When the effective resistance between two circuit blocks decreases, noise coupling through the power network increases, which can now be quantified by the effective resistance described in this brief. The effective resistance is also used to determine the coverage and commute times of a random walk in a graph [15]. In an undirected resistive graph, the effective resistance is used to determine the effective chemical distance between bonds, as in [16]. The effective resistance is also used in distributive control and estimation such as synchronization and localization of sensor networks [17].

Venezian [18] developed a closed-form expression of the resistance of a uniform mesh where the vertical and horizontal unit resistances are the same. The work described in this brief is inspired by [18], where the effective resistance is generalized for nonisotropic meshes with different vertical and horizontal unit resistances. To determine the effective resistance between nodes $n_{x_{1}, y_{1}}$ and $n_{x_{2}, y_{1}}$, where $x_{1}, x_{2}$, and $y_{1}$ are, respectively, the horizontal and vertical coordinates of the nodes within an infinite mesh, as shown in Fig. 2(a), the principal of superposition is applied in two steps [18], [19]. First, current $I$ is introduced at $n_{x_{1}, y_{1}}$ and exits the grid at the boundaries (i.e., at infinity), as illustrated in Fig. 2(b). The current from $n_{x_{1}, y_{1}}$ to the adjacent nodes is determined by the resistance between $n_{x_{1}, y_{1}}$ and the adjacent nodes. When the mesh is uniform, the currents from $\left(x_{1}, y_{1}\right)$ to the adjacent nodes are symmetric and $I / 4$. Second, current $I$ is introduced at infinity and exits the grid at $n_{x_{2}, y_{1}}$, as depicted in Fig. 2(c). The current from the nodes adjacent to $n_{x_{2}, y_{1}}$ is similarly determined. When the mesh is uniform, the currents from the adjacent nodes of $\left(x_{1}, y_{1}\right)$ to $\left(x_{1}, y_{1}\right)$ are again symmetric and $I / 4$. By applying superposition in these two steps, current $I$ is modeled as entering the grid from $n_{x_{1}, y_{1}}$ and exiting the grid at $n_{x_{2}, y_{1}}$, as shown in Fig. 2(d). This current is the sum of the currents in the first and second steps of the superposition process, which is therefore $I / 2$. The voltage difference divided by the current provides the effective resistance. The effective resistance between $n_{x_{1}, y_{1}}$ and $n_{x_{2}, y_{1}}$ within a uniform mesh is therefore

$$
R_{\mathrm{eff}}=2\left(V_{x_{1}, y_{1}}-V_{x_{2}, y_{1}}\right) / I .
$$




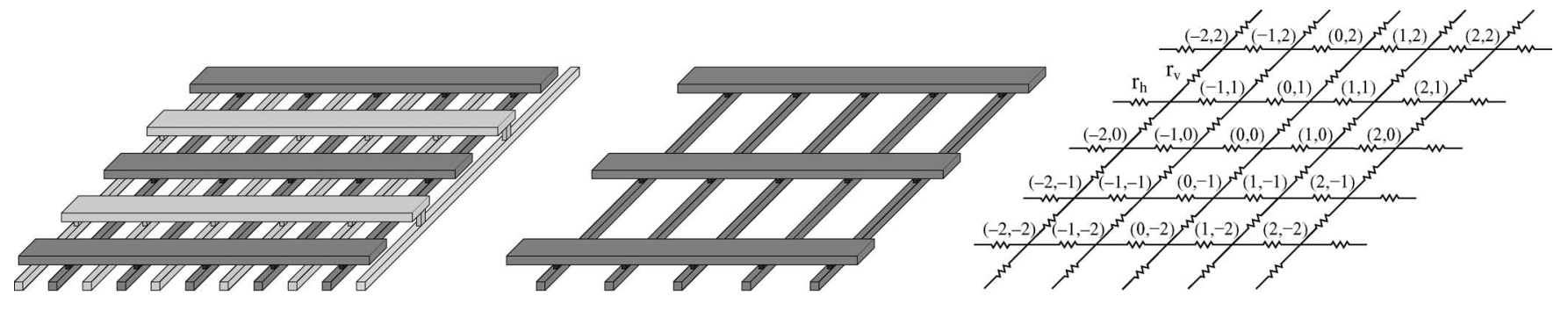

(a)

(b)

(c)

Fig. 1. Two layer orthogonal metal lines connected with vias; (a) two layer power and ground distribution network where the power and ground lines are illustrated, respectively, with dark and light gray, (b) a two layer power distribution network only, and (c) a resistive mesh model of the power distribution network.

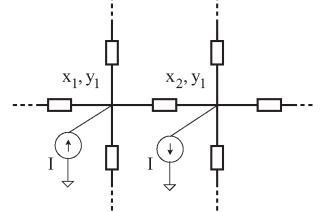

(a)

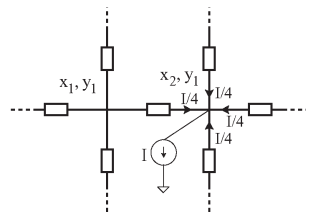

(c)

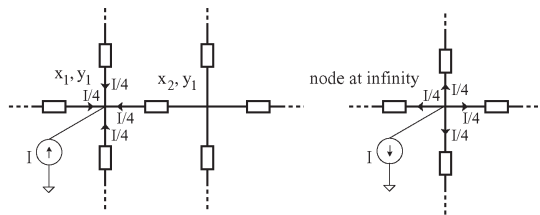

(b)

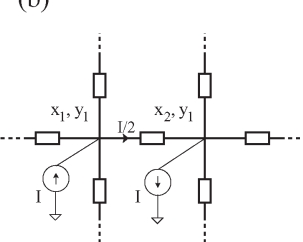

(d)
Fig. 2. In an infinite mesh structure, (a) current source $I$ is connected to $\left(x_{1}, y_{1}\right)$, current load $I$ is connected to $\left(x_{1}, y_{2}\right)$, and the effective resistance between these adjacent nodes is determined by applying the principle of superposition in two steps. In the first step, (b) the load current is moved to a node at infinity, and in the second step, (c) the source current is moved to a node at infinity. The current profiles for these two cases are obtained, (d) the current source and load are moved to the original positions, and the current during the two superposition steps is summed to determine the effective resistance.

A similar analysis is performed for a nonisotropic mesh structure with different horizontal and vertical resistances to determine closed-form expressions for the effective resistance between two arbitrary nodes.

The brief is organized as follows. In Section II, Kirchhoff's current law is revisited to determine the voltages and currents at a particular node in terms of the neighboring node voltages and resistances. In Section III, inhomogeneous differential equations are applied, where separation of variables is used to determine the node voltages. The effective resistance between two arbitrary intersections and the corresponding closed-form expressions are described, respectively, in Sections IV and V. The accuracy of the effective resistance model is discussed in Section VI. The brief is summarized in Section VII followed by an Appendix, describing the derivation of the closed-form expression for the effective resistance.

\section{KiRChHOFF's CURRENT LAW REVISITED}

The mesh circuit model considered in this brief is shown in Fig. 1(c) with horizontal $\left(r_{h}\right)$ and vertical $\left(r_{v}\right)$ resistors. The voltage at node $n_{x, y}$ is $V_{x, y}$, and the current from $n_{x, y}$ to ground is $I_{x, y}$. When a current source is connected to $n_{x, y}$, $I_{x, y}=I$. Alternatively, when no current source is connected to $n_{x, y}, I_{x, y}=0$.

The current load at an arbitrary node $n_{x, y}$ can be written in terms of the sum of the current from the four adjacent nodes as

$$
\begin{aligned}
I_{x, y}=\frac{V_{x, y}-V_{x, y+1}}{r_{v}} & +\frac{V_{x, y}-V_{x, y-1}}{r_{v}} \\
& +\frac{V_{x, y}-V_{x+1, y}}{r_{h}}+\frac{V_{x, y}-V_{x-1, y}}{r_{h}} .
\end{aligned}
$$

The vertical resistance between adjacent nodes is $r$, and the horizontal resistance between adjacent nodes is $k * r$, where $k$ is number $0<k<\infty$, as

$$
\begin{aligned}
& r_{v}=r \\
& r_{h}=k * r .
\end{aligned}
$$

When $I_{x, y}=0$, the voltage at $n_{x, y}$ is

$$
V_{x, y}=\frac{k V_{x, y+1}+k V_{x, y-1}+V_{x+1, y}+V_{x-1, y}}{2 k+2} .
$$

When a current source is connected to $n_{x, y}$, this current can be described in terms of the adjacent node voltages and corresponding resistors as

$$
I_{x, y}=\frac{(2 k+2) V_{x, y}-\left(k V_{x, y+1}+k V_{x, y-1}+V_{x+1, y}+V_{x-1, y}\right)}{k r} \text {. }
$$

\section{SEPARATion OF VARiables}

The difference equations (5) and (6) can be solved by applying separation of variables [18]. A solution for (5) is

$$
V_{x, y}=e^{x \alpha+j y \beta} \text {. }
$$

By substituting (7) into (5), (5) can be written as

$$
\begin{aligned}
(2 k+2) e^{x \alpha+j y \beta} & =e^{x \alpha+j y \beta}\left(k e^{j \beta}+k e^{-j \beta}+e^{\alpha}+e^{-\alpha}\right) \\
2 k+2 & =k\left(e^{j \beta}+e^{-j \beta}\right)+\left(e^{\alpha}+e^{-\alpha}\right)
\end{aligned}
$$

Using the cosine and sine properties, (9) is

$$
k+1=k \cos \beta+\cosh \alpha .
$$

When a current source is connected to $n_{0,0}$ and is assumed to exit the system at infinity, the following equations are satisfied due to the symmetry of the mesh structure as follows:

$$
V_{x, y}=V_{-x, y}=V_{x,-y}=V_{-x,-y} .
$$

One possible solution to (11) is

$$
V_{x, y}=e^{-|x| \alpha} \cos y \beta \text {. }
$$

The currents can be described in terms of these voltages. By substituting $x=y=0$ into (6), current $i_{0,0}$ at $n_{0,0}$ becomes

$$
i_{0,0}=\frac{(2 k+2) V_{0,0}-k V_{0,1}-k V_{0,-1}-V_{1,0}-V_{-1,0}}{k r} .
$$


By substituting (12) into (13), current $I$ at $n_{0,0}$ becomes

$$
i_{0,0}=2\left(k+1-k \cos \beta-e^{-\alpha}\right) / k r .
$$

By substituting (10) into (14), the current at $n_{0,0}$ becomes

$$
i_{0,0}=\left(2 \cosh \alpha-2 e^{-\alpha}\right) / k r .
$$

By using identities $\cosh x=1 / 2\left(e^{x}+e^{-x}\right)$ and $\sinh x=$ $1 / 2\left(e^{x}-e^{-x}\right)$ from Euler's formula [20], the current expression $i_{0,0}$ becomes

$$
i_{0,0}=2 \sinh \alpha / k r .
$$

Similarly, when $y \neq 0$, current $i_{0, y}$ at $n_{0, y}$ becomes

$$
i_{0, y}=\frac{(2 k+2) V_{0, y}-k V_{0, y+1}-k V_{0, y-1}-V_{1, y}-V_{-1, y}}{k r}
$$

and by substituting (10) into (17), the current can be rewritten as

$$
\begin{array}{r}
i_{0, y}=\left((2 k+2) \cos y \beta-e^{-\alpha} \cos y \beta-e^{-\alpha} \cos y \beta\right. \\
-k \cos (y+1) \beta-k \cos (y-1) \beta) / k r .
\end{array}
$$

After applying certain trigonometric identities and simplifications

$$
i_{0, y}=\left(\left(2 k+2-2 e^{-\alpha}\right) \cos y \beta-2 k \cos y \beta \cos \beta\right) / k r .
$$

The current $i_{0, y}$ at $n_{0, y}$ is

$$
i_{0, y}=2\left(k+1-e^{-\alpha}-k \cos \beta\right) \cos y \beta / k r .
$$

By substituting (10) into (20) and applying Euler's formula, the current at $n_{0, y}$ becomes

$$
i_{0, y}=\frac{2 \sinh \alpha \cos y \beta}{k r} .
$$

\section{Effective Resistance Between Two Nodes}

The voltage at $n_{x, y}$ is a function of $\alpha$ and $\beta$ where the relationship between these two parameters in (10) is in terms of $k$. The voltage at an arbitrary node $n_{x, y}$ is the sum of all $\beta$ values as follows:

$$
V_{x, y}=\int_{0}^{\pi} F(\beta) v_{x, y}(\beta) d \beta
$$

where $F(\beta)$ is a function that satisfies a current source at $n_{0,0}$ and satisfies no current source at $n_{0, y}$ when $y \neq 0$. Thus, all of the current sources other than at $n_{0,0}$ are effectively eliminated [18]. The corresponding current at $n_{x, y}$ is

$$
I_{x, y}=\int_{0}^{\pi} F(\beta) i_{x, y}(\beta) d \beta .
$$

The current at $n_{0,0}$, by substituting (16) into (23), becomes

$$
I_{0,0}=\int_{0}^{\pi} F(\beta) \frac{2 \sinh \alpha}{k} d \beta
$$

and the current at $n_{0, y}$, by substituting (21) into (23), becomes

$$
I_{0, n}=\int_{0}^{\pi} F(\beta) \frac{2 \sinh \alpha \cos y \beta}{k} d \beta .
$$

From inspection, $F(\beta)$ is

$$
F(\beta)=\frac{k I r}{2 \pi \sinh \alpha}
$$

to satisfy (22) when only one current source located at $n_{0,0}$ is present within the mesh. By substituting (26) and (12) into (22), the voltage at $n_{x, y}$ becomes

$$
V_{x, y}=\frac{k I r}{2 \pi} \int_{0}^{\pi} \frac{e^{-|x| \alpha} \cos y \beta}{\sinh \alpha} d \beta .
$$

\section{Closed-Form EXPRESSION OF THE EFFECTIVE RESISTANCE}

The effective resistance of a mesh between $n_{0,0}$ and $n_{x, y}$ is

$$
R_{x, y}=2\left(V_{0,0}-V_{x, y}\right) / I
$$

as discussed in Section I. By substituting (27) into (28), the effective resistance between $n_{0,0}$ and $n_{x, y}$ becomes

$$
R_{x, y}=\frac{k r}{\pi} \int_{0}^{\pi} \frac{\left(2-e^{-|x| \alpha} \cos y \beta\right)}{\sinh \alpha} d \beta .
$$

$R_{x, y}$ is solved by dividing the integral into two, and by writing (29) as a sum of two integrals, $R_{x, y} / r=R_{1(x, y)}+R_{2(x, y)}$, i.e.,

$$
\begin{aligned}
R_{x, y} / r & =\frac{\sqrt{k}}{\pi} \int_{0}^{\pi} \frac{\left(1-e^{-x \sqrt{k}|\beta|} \cos y \beta\right)}{\beta} d \beta \\
& +\frac{k}{\pi} \int_{0}^{\pi}\left[\frac{1}{\sqrt{(k+1-k \cos \beta)^{2}-1}}-\frac{1}{\beta \sqrt{k}}\right] d \beta .
\end{aligned}
$$

The first integral $R_{1(x, y)}$ is rewritten in terms of the exponential integral $\operatorname{Ein}(z)[20]$, as follows:

$$
\operatorname{Ein}(z)=\int_{0}^{z} \frac{1-e^{-t}}{t} d \beta
$$

and $R_{1(x, y)}$, i.e.,

$$
R_{1(x, y)}=(1 / \pi k) \operatorname{Re}\{\operatorname{Ein}[\pi(\sqrt{k} x+)]\}
$$

is numerically solved, and $R_{1(x, y)}$ is

$$
R_{1(x, y)}=\frac{\sqrt{k}}{2 \pi}\left[\ln \left(x^{2}+k y^{2}\right)+2(0.57721+\ln \pi)\right]
$$

while the second integral $R_{2(x, y)}$ is determined assuming $k=$ $n+\epsilon$, as follows:

$R_{2(x, y)}=\frac{k}{\pi} \int_{0}^{\pi}\left(\left((n+1-n \cos \beta)^{2}-1\right)^{-1 / 2}-\frac{1}{\beta \sqrt{n}}\right) d \beta$

$+\frac{k}{\pi} \int_{0}^{\pi}\left(-\epsilon \frac{(1-\cos \beta)(n+1-n \cos \beta)}{\left((n+1-n \cos \beta)^{2}-1\right)^{3 / 2}}+\frac{\epsilon}{2 \beta n \sqrt{n}}\right) d \beta$.

A derivation of (34) is provided in the Appendix. The effective resistance between any two arbitrary nodes $R_{x, y}$ within a mesh when $k$ approaches a different constant is listed in Table I. For instance, the effective resistance when $k \rightarrow 1$ is

$$
\begin{aligned}
R_{x, y} / r & =\frac{\sqrt{k}}{2 \pi}\left[\ln \left(x^{2}+k y^{2}\right)+3.44388\right] \\
& -0.033425 k-0.0629 k(k-1), \quad \text { for } k \rightarrow 1 .
\end{aligned}
$$


TABLE I

Closed-Form EXPRESSIONS FOR $R_{1(x, y)}$ AND $R_{2(x, y)}$ WHeRE $R_{(x, y)} / r=R_{1(x, y)}+R_{2(x, y)}$ When $k$ APproaches A Constant

\begin{tabular}{|l||c|c|c|}
\hline$k \rightarrow$ & $R_{1(x, y)}$ & $R_{2(x, y)}$ & eq. \# \\
\hline \hline 1 & $\frac{\sqrt{k}}{2 \pi}\left[\ln \left(x^{2}+k y^{2}\right)+3.4439\right]$ & $-0.0334 k-0.0629 k(k-1)$ & $(35)$ \\
\hline 2 & $\frac{\sqrt{k}}{2 \pi}\left[\ln \left(x^{2}+k y^{2}\right)+3.4439\right]$ & $-0.0692 k-0.0202 k(k-2)$ & $(44)$ \\
\hline 3 & $\frac{\sqrt{k}}{2 \pi}\left[\ln \left(x^{2}+k y^{2}\right)+3.4439\right]$ & $-0.0829 k-0.0093 k(k-3)$ & $(45)$ \\
\hline 4 & $\frac{\sqrt{k}}{2 \pi}\left[\ln \left(x^{2}+k y^{2}\right)+3.4439\right]$ & $-0.0896 k-0.0047 k(k-4)$ & $(46)$ \\
\hline 5 & $\frac{\sqrt{k}}{2 \pi}\left[\ln \left(x^{2}+k y^{2}\right)+3.4439\right]$ & $-0.0932 k-0.0026 k(k-5)$ & $(47)$ \\
\hline 10 & $\frac{\sqrt{k}}{2 \pi}\left[\ln \left(x^{2}+k y^{2}\right)+3.4439\right]$ & $-0.0964 k+0.00021 k(k-10)$ & $(48)$ \\
\hline 100 & $\frac{\sqrt{k}}{2 \pi}\left[\ln \left(x^{2}+k y^{2}\right)+3.4439\right]$ & $-0.0657 k+0.00016 k(k-100)$ & $(49)$ \\
\hline
\end{tabular}

TABLE II

ACCURACY OF THE Closed-Form SOLUtion For the EFFeCtive RESISTANCE WHEN $r_{v}=1 \Omega, r_{h}=k \Omega$, AND $r_{\mathrm{via}}=l \Omega$

\begin{tabular}{|c|c|c|c|c|c|c|}
\hline & \multirow{3}{*}{$\begin{array}{c}x=0 \\
y=1 \\
0.5147\end{array}$} & \multirow{3}{*}{$\begin{array}{c}x=1 \\
y=0 \\
0.5147\end{array}$} & \multirow{3}{*}{$\begin{array}{c}x=10 \\
y=0 \\
1.2476\end{array}$} & \multirow{3}{*}{$\begin{array}{l}x=10 \\
y=10 \\
1.3580\end{array}$} \\
\hline & & & & & & \\
\hline \multirow{7}{*}{$\mathrm{k}=1$} & & (35) & & & & \\
\hline & \multirow{2}{*}{$1=0$} & SPICE & 0.5 & 0.5 & 1.2480 & 1.3580 \\
\hline & & Error & $3 \%$ & $3 \%$ & $0 \%$ & $0 \%$ \\
\hline & \multirow{2}{*}{$1=0.01$} & SPICE & 0.5033 & 0.5033 & 1.2546 & 1.3642 \\
\hline & & Error & $2.2 \%$ & $2.2 \%$ & $0.6 \%$ & $0.5 \%$ \\
\hline & \multirow{2}{*}{$1=0.05$} & SPICE & 0.5167 & 0.5167 & 1.2819 & 1.393 \\
\hline & & Error & $0.4 \%$ & $0.4 \%$ & $2.7 \%$ & $2.6 \%$ \\
\hline \multirow{7}{*}{$\mathrm{k}=2$} & & (44) & 0.6367 & 0.7928 & 1.6733 & 1.9205 \\
\hline & \multirow{2}{*}{$1=0$} & SPICE & 0.6082 & 0.7838 & 1.6737 & 1.9206 \\
\hline & & Error & $4.7 \%$ & $1.1 \%$ & $0 \%$ & $0 \%$ \\
\hline & \multirow{2}{*}{$1=0.01$} & SPICE & 0.6098 & 0.7886 & 1.6775 & 1.9299 \\
\hline & & Error & $4.2 \%$ & $0.6 \%$ & $0.3 \%$ & $0.5 \%$ \\
\hline & \multirow{2}{*}{$1=0.05$} & SPICE & 0.6168 & 0.8122 & 1.6969 & 1.9677 \\
\hline & & Error & $3.1 \%$ & $3.6 \%$ & $1.4 \%$ & $2.5 \%$ \\
\hline \multirow{7}{*}{$\mathrm{k}=5$} & & (47) & 0.7596 & 1.3324 & 2.399 & 3.0362 \\
\hline & \multirow{2}{*}{$1=0$} & SPICE & 0.7322 & 1.3391 & 2.399 & 3.0361 \\
\hline & & Error & $3.7 \%$ & $1.1 \%$ & $0 \%$ & $0 \%$ \\
\hline & \multirow{2}{*}{$1=0.01$} & SPICE & 0.7331 & 1.3436 & 2.4013 & 3.0485 \\
\hline & & Error & $3.5 \%$ & $0.8 \%$ & $0.1 \%$ & $0.4 \%$ \\
\hline & \multirow{2}{*}{$1=0.05$} & SPICE & 0.7355 & 1.382 & 2.4130 & 3.0995 \\
\hline & & Error & $3.2 \%$ & $3.7 \%$ & $0.6 \%$ & $2.1 \%$ \\
\hline \multirow{7}{*}{$\mathrm{k}=10$} & & (48) & 0.769 & 1.928 & 3.087 & 4.294 \\
\hline & \multirow{2}{*}{$1=0$} & SPICE & 0.805 & 1.952 & 3.088 & 4.294 \\
\hline & & Error & $4.7 \%$ & $1.2 \%$ & $0 \%$ & $0 \%$ \\
\hline & \multirow{2}{*}{$1=0.01$} & SPICE & 0.8057 & 1.9464 & 3.0887 & 4.307 \\
\hline & & Error & $4.8 \%$ & $1 \%$ & $0.1 \%$ & $0.3 \%$ \\
\hline & \multirow{2}{*}{$1=0.05$} & SPICE & 0.8066 & 1.9958 & 3.0972 & 4.3684 \\
\hline & & Error & $4.9 \%$ & $3.5 \%$ & $0.3 \%$ & $1.7 \%$ \\
\hline
\end{tabular}

\section{EXPERIMENTAL RESULTS}

The accuracy of the proposed effective resistance model is compared with the exact solution (29) in Table II. Although the via resistance $r_{\text {via }}$ connecting orthogonal metal layers is neglected in the proposed effective resistance model, for practical values of $r_{\text {via }}$ (i.e., when $r_{\text {via }}$ is between zero and $5 \%$ of the horizontal or vertical resistance [21]), the proposed effective resistance model is in good agreement with the experimental results. The via resistance $r_{\text {via }}$ is modeled as $r_{\text {via }}=l \cdot r_{v}$. The maximum error is less than $5 \%$ for $1<k<10$ and $0<l<$ 0.05 . The error is maximum when the distance between the two nodes is smallest, and the error decreases with greater separation between the nodes of interest if $r_{\text {via }}$ is zero. $r_{\text {via }}$ is neglected in the expressions; the approximation error in the proposed expressions converges to zero with greater separation between the nodes of interest. When $r_{\text {via }}$ is nonzero, the error exhibits a nonmonotonic behavior (i.e. the error does not necessarily decrease with greater separation between the nodes of interest).
TABLE III

ERROR INDUCED BY THE INFINITE GRID APPROXIMATION FOR POWER GRIDS WITH DIFFERENT SizES

\begin{tabular}{|c|c|c|c|c|c|}
\hline & & $\begin{array}{l}x=0 \\
y=1\end{array}$ & $\begin{array}{l}x=2 \\
y=3\end{array}$ & $\begin{array}{c}x=10 \\
y=0\end{array}$ & $\begin{array}{c}x=10 \\
y=10\end{array}$ \\
\hline & (35) & 0.5147 & 0.924 & 1.2476 & 1.358 \\
\hline \multirow{2}{*}{$20 \times 20$} & SPICE & 0.5015 & 0.9425 & 1.3838 & 1.665 \\
\hline & Error & $2.6 \%$ & $2 \%$ & $10.9 \%$ & $22.6 \%$ \\
\hline \multirow{2}{*}{$30 \times 30$} & SPICE & 0.5006 & 0.9324 & 1.3079 & 1.486 \\
\hline & Error & $2.8 \%$ & $0.9 \%$ & $4.8 \%$ & $9.4 \%$ \\
\hline \multirow{2}{*}{$40 \times 40$} & SPICE & 0.5004 & 0.929 & 1.2815 & 1.4284 \\
\hline & Error & $2.9 \%$ & $0.5 \%$ & $2.7 \%$ & $5.2 \%$ \\
\hline \multirow{2}{*}{$80 \times 80$} & SPICE & 0.5 & 0.925 & 1.252 & 1.367 \\
\hline & Error & $3 \%$ & $0.1 \%$ & $0.4 \%$ & $0.7 \%$ \\
\hline
\end{tabular}

Practical mesh structures have finite dimensions. Since an infinite mesh is assumed in the development of these expressions, the error of the proposed expressions is compared with four differently sized mesh structures (see Table III where $k=1$ and $l=0$ ). With increasing separation between the nodes of interest, the error originating from the infinite grid assumption naturally increases. Additionally, the error increases when both nodes of interest are not at the center of the finite grid. In Table III, both nodes of interest $(x, y)=(2,3)$ and $(x, y)=$ $(10,10)$ are not at the center of the grid. The error is less than $3 \%$ when the nodes of interest are twenty lines from the boundary.

\section{CONCLUSION}

A closed-form expression for the effective resistance of a two layer mesh structure is presented in this brief. The unit resistance of the horizontal and vertical metal lines within a power grid is often different in adjacent orthogonal metal layers due to the difference in the width and thickness of these metal lines. The closed-form expression presented in this brief uses parameter $k$ to model the ratio of the horizontal and vertical resistances. The closed-form expression provides a fast and accurate solution to the effective resistance of a two layer mesh, which can be used to solve a variety of problems found in different disciplines. Examples include $I R$ voltage drop analysis of integrated circuits, synchronization and localization of sensor networks, the effective chemical distance between bonds, metal mesh interference filters in terahertz physics, and the commute and cover times of undirected graphs.

\section{APPENDIX}

$R_{2}(x, y)$ is simplified to obtain a numerical solution similar to $R_{1}(x, y)$. Multiple numerical solutions exist for different values of $k$. To obtain a general solution of $R_{2}(x, y)$ for all possible values of $k, k$ is expanded when approaching a positive real number $n$. In this Appendix, the second part of the integral in (30) is simplified by applying a Taylor series expansion when $k \rightarrow n+\epsilon$, where $\epsilon \ll 1$ (i.e., when $k$ approaches $n$ ) and certain trigonometric identities. From (30), $R_{2(x, y)}$ is

$$
R_{2(x, y)}=\frac{k}{\pi} \int_{0}^{\pi}\left[\frac{1}{\sqrt{(k+1-k \cos \beta)^{2}-1}}-\frac{1}{\beta \sqrt{k}}\right] d \beta .
$$

By substituting $(1+\epsilon)^{m} \approx 1+m \epsilon$ multiple times into (36), $R_{2(x, y)}$ is simplified to (37)-(40), as shown at the top of the next page. 


$$
\begin{aligned}
& R_{2(x, y)}=\frac{k}{\pi} \int_{0}^{\pi}\left(\left\{(n+\epsilon+1-(n+\epsilon) \cos \beta)^{2}-1\right\}^{-1 / 2}-\frac{1}{\beta}(n+\epsilon)^{-1 / 2}\right) d \beta \\
& R_{2(x, y)}=\frac{k}{\pi} \int_{0}^{\pi}\left(\left\{(n+1-n \cos \beta)^{2}\left(1+\epsilon \frac{1-\cos \beta}{n+1-n \cos \beta}\right)^{2}-1\right\}^{-1 / 2}-\frac{1}{\beta \sqrt{n}}\left(1-\epsilon \frac{1}{2 n}\right)\right) d \beta \\
& R_{2(x, y)}=\frac{k}{\pi} \int_{0}^{\pi}\left(\left((n+1-n \cos \beta)^{2}-1+2 \epsilon(1-\cos \beta)(n+1-n \cos \beta)\right)^{-1 / 2}-\frac{1}{\beta \sqrt{n}}+\epsilon \frac{1}{2 n \sqrt{n} \beta}\right) d \beta \\
& R_{2(x, y)}=\frac{k}{\pi} \int_{0}^{\pi}\left(\left((n+1-n \cos \beta)^{2}-1\right)^{-1 / 2}\left(1-\epsilon \frac{(1-\cos \beta)(n+1-n \cos \beta)}{(n+1-n \cos \beta)^{2}-1}\right)-\frac{1}{\beta \sqrt{n}}+\epsilon \frac{1}{2 n \sqrt{n} \beta}\right) d \beta
\end{aligned}
$$

$R_{2(x, y)}$ is grouped into two parts as follows:

$$
\begin{aligned}
& R_{2(x, y)}=\frac{k}{\pi} \int_{0}^{\pi}\left(\left((n+1-n \cos \beta)^{2}-1\right)^{-1 / 2}-\frac{1}{\beta \sqrt{n}}\right) d \beta \\
& +\frac{k}{\pi} \int_{0}^{\pi}\left(-\epsilon \frac{(1-\cos \beta)(n+1-n \cos \beta)}{\left((n+1-n \cos \beta)^{2}-1\right)^{3 / 2}}+\frac{\epsilon}{2 \beta n \sqrt{n}}\right) d \beta .
\end{aligned}
$$

$R_{2(x, y)}$ can be numerically determined by assigning $n$ to a constant. For instance, when $k \rightarrow 1$ (i.e., $n=1$ ), the first and second parts of (41) are numerically determined by, respectively, assigning $n=1$ and substituting $\epsilon=k-1 . R_{2(x, y)}$ becomes

$$
\begin{aligned}
& R_{2(x, y)}=-0.033425 k \\
& \quad-\frac{k(k-1)}{\pi} \int_{0}^{\pi}\left(\frac{(1-\cos \beta)(2-\cos \beta)}{\left((2-\cos \beta)^{2}-1\right)^{3 / 2}}-\frac{1}{2 \beta}\right) d \beta .
\end{aligned}
$$

The second integral is numerically solved, and the closedform expression for $R_{2(x, y)}$ when $k \rightarrow 1$ is

$$
R_{2(x, y)}=-0.033425 k-0.0629 k(k-1) .
$$

When $k$ approaches another constant, (41) is similarly determined. Closed-form approximations for $R_{1(x, y)}$ and $R_{2(x, y)}$ are listed in Table I for different values of $n$, where the effective resistance $R_{x, y}=R_{1(x, y)}+R_{2(x, y)}$.

\section{ACKNOWLEDGMENT}

The authors would like to thank M. Yönaç for his valuable suggestions.

\section{REFERENCES}

[1] R. Jakushokas, M. Popovich, A. V. Mezhiba, S. Kose, and E. G. Friedman, Power Distribution Networks With On-Chip Decoupling Capacitors, 2nd ed. New York: Springer-Verlag, 2011.

[2] J. N. Kozhaya, S. R. Nassif, and F. N. Najm, "A multigrid-like technique for power grid analysis," IEEE Trans. Comput.-Aided Des. Integr. Circuits Syst., vol. 21, no. 10, pp. 1148-1160, Oct. 2002.

[3] L. Zlydina and Y. Yagil, "3D power grid modeling," in Proc. IEEE Int. Conf. Electron., Circuits Syst., Dec. 2004, pp. 129-132.
[4] J. Singh and S. S. Sapatnekar, "Partition-based algorithm for power grid design using locality," IEEE Trans. Comput.-Aided Des. Integr. Circuits Syst., vol. 25, no. 4, pp. 664-677, Apr. 2006.

[5] K. Lee and A. Barber, "Modeling and analysis of multichip module power supply planes," IEEE Trans. Compon., Packag., Manuf. Technol. B, Adv. Packag., vol. 18, no. 4, pp. 628-639, Nov. 1995.

[6] S. Kose and E. G. Friedman, "Fast algorithms for IR voltage drop analysis exploiting locality," in Proc. IEEE/ACM Des. Autom. Conf., Jun. 2011, pp. 996-1001.

[7] Y. Ogasahara, M. Hashimoto, T. Kanamoto, and T. Onoye, "Measurement of supply noise suppression by substrate and deep N-well in $90 \mathrm{~nm}$ process," in Proc. IEEE Asian Solid-State Circuits Conf., Nov. 2008, pp. 397-400.

[8] Z. Shiyou, K. Roy, and C.-K. Koh, "Decoupling capacitance allocation and its application to power-supply noise-aware floorplanning," IEEE Trans. Comput.-Aided Des. Integr. Circuits Syst., vol. 21, no. 1, pp. 81-92, Jan. 2002.

[9] E. Wong, J. R. Minz, and S. K. Lim, "Decoupling-capacitor planning and sizing for noise and leakage reduction," IEEE Trans. Comput.-Aided Design Integr. Circuits Syst., vol. 26, no. 11, pp. 2023-2034, Nov. 2007.

[10] M. Popovich, M. Sotman, A. Kolodny, and E. G. Friedman, "Effective radii of on-chip decoupling capacitors," IEEE Trans. Very Large Scale Integr. (VLSI) Circuits, vol. 16, no. 7, pp. 894-907, Jul. 2008.

[11] S. Köse and E. G. Friedman, "Distributed power network co-design with on-chip power supplies and decoupling capacitors," in Proc. Workshop Syst. Level Interconnect Prediction, Jun. 2011, pp. 1-5.

[12] J. Rommes and W. H. A. Schilders, "Efficient methods for large resistor networks," IEEE Trans. Comput.-Aided Design Integr. Circuits Syst., vol. 29, no. 1, pp. 28-39, Jan. 2010.

[13] R. Helinski and J. Plusquellic, "Measuring power distribution system resistance variations," IEEE Trans. Semicond. Manuf., vol. 21, no. 3, pp. 444-453, Aug. 2008.

[14] A. Todri, M. Marek-Sadowska, F. Maire, and C. Matheron, "A study of decoupling capacitor effectiveness in power and ground grid networks," in Proc. IEEE Int. Symp. Quality Electron. Des., Mar. 2009, pp. 653-658.

[15] A. K. Chandra, P. Raghavan, W. L. Ruzzo, and R. Smolensky, "The electrical resistance of a graph captures its commute and cover times," in Proc. Annu. ACM Symp. Theory Comput., 1989, pp. 574-586.

[16] D. J. Klein and M. Randi, "Resistance distance," J. Math. Chem., vol. 12, no. 1, pp. 81-95, Dec. 1993.

[17] P. Barooah and J. P. Hespanha, "Graph effective resistance and distributed control: Spectral properties and applications," in Proc. IEEE Conf. Decision Control, Dec. 2006, pp. 3479-3485.

[18] G. Venezian, "On the resistance between two points on a grid," Amer. J. Phys., vol. 62, no. 11, pp. 1000-1004, Nov. 1994.

[19] C. R. Paul, Analysis of Linear Circuits. New York: McGraw-Hill, 1989.

[20] M. Abramowitz and I. A. Stegun, Handbook of Mathematical Functions with Formulas, Graphs, and Mathematical Tables. New York: Dover, 1972.

[21] S. R. Nassif, "Power grid analysis benchmarks," in Proc. IEEE/ACM Asia South Pacific Design Autom. Conf., Jan. 2008, pp. 376-381. 